\title{
BMJ Open Building motivation to participate in a quality improvement collaborative in NHS hospital trusts in Southeast England: a qualitative participatory evaluation
}

\author{
Mirza Lalani, ${ }^{1}$ Kate Hall, ${ }^{2}$ Mirek Skrypak, ${ }^{2}$ Chris Laing, ${ }^{3}$ John Welch, ${ }^{4}$ Peter Toohey, ${ }^{2}$ \\ Sarah Seaholme, ${ }^{2}$ Thomas Weijburg, ${ }^{2}$ Laura Eyre, ${ }^{1}$ Martin Marshall ${ }^{1}$
}

To cite: Lalani $\mathrm{M}$, Hall $\mathrm{K}$, Skrypak M, et al. Building motivation to participate in a quality improvement collaborative in NHS hospital trusts in Southeast England: a qualitative participatory evaluation. BMJ Open 2018;8:e020930. doi:10.1136/ bmjopen-2017-020930

- Prepublication history for this paper is available online. To view these files, please visit the journal online (http://dx.doi org/10.1136/bmjopen-2017020930).

Received 5 December 2017 Revised 8 February 2018 Accepted 6 March 2018

\section{Check for updates}

${ }^{1}$ Research Department of Primary Care and Population Health, University College London, London, UK ${ }^{2}$ UCLPartners, London, UK ${ }^{3}$ The Royal Free London NHS Foundation Trust, London, UK ${ }^{4}$ University College London Hospitals NHS Foundation Trust, London, UK

Correspondence to

Mirza Lalani; m.lalani@ucl.ac.uk

\section{ABSTRACT}

Objectives This study explores the barriers and facilitators that impact on the motivation of practitioners to participate in a quality improvement collaborative.

Design A qualitative and formative evaluation using a participatory approach, the researcher-in-residence model which embraces the concept of 'coproducing' knowledge between researchers and practitioners using a range of research methods such as participant observation, interviews and documentary analysis. The design, creation and application of newly generated evidence are facilitated by the researcher through negotiation and compromise with team members.

Participants Senior and middle managers, doctors and nurses.

Setting Two hospitals in Southeast England participating in a Patient Safety Improvement Collaborative and the facilitator (host) of the collaborative, based in Central London.

Results The evaluation has revealed facilitators and barriers to motivation categorised under two main themes: (1) inherent motivation and (2) factors that influence motivation, interorganisational and intraorganisational features as well as external factors. Facilitators included collaborative 'champions,' individuals who drove the quality improvement agenda at a local level, raising awareness and inspiring colleagues. The collaborative itself acted as a facilitator, promoting shared learning as well as building motivation for participation. A key barrier was the lack of board engagement in the participating National Health Service organisations which may have affected motivation among front-line staff.

Conclusions Collaboratives maybe an important way of engaging practitioners in quality improvement initiatives.

This study highlights that despite a challenging healthcare environment in the UK, there remains motivation among individuals to participate in quality improvement programmes as they recognise that improvement approaches may facilitate positive change in local clinical processes and systems. Collaboratives can harness this individual motivation to facilitate spread and adoption of improvement methodology and build engagement across their membership.

\section{Strengths and limitations of this study}

- The use of the in-residence model and a formative and qualitative approach to evaluation is novel in the context of a quality improvement collaborative. This approach develops our understanding of the factors affecting motivation of clinicians and managers to engage with quality improvement initiatives.

- A limitation of the study is that the researcher was only embedded in two hospital trusts, raising questions of generalisability; nevertheless, we believe that the concepts generated are likely to be transferrable to the rest of the improvement collaborative and other similar initiatives.

- Maintaining objectivity in participatory research can be challenging as the researcher's fellow team members are also participants in the evaluation. This was minimised by the researcher regularly discussing findings with independent academic colleagues to obtain different perspectives while also recognising the importance of including individuals familiar with the programme, providing a context for findings and enabling learning through the evaluation process.

\section{BACKGROUND}

Quality improvement collaboratives in healthcare bring together groups of health professionals, managers and support staff either within an organisation or from several organisations, to work on a common purpose, with the goal of improving health services. ${ }^{1}$ Improvement collaboratives have become increasingly popular in the UK, with the National Health Service (NHS) promoting them as a mechanism for change across the healthcare system involving several types of organisations including general practitioner surgeries, hospitals and care homes. ${ }^{2-4}$ Improvement collaboratives frequently follow a Breakthrough Series approach ${ }^{5}$ supporting 
organisations to close the gap between good care and usual practice in a short time period (6-18 months). During this time, teams meet to share learning on a topic (learning sessions), to understand how to make improvements, to implement and test these improvements, and to share their progress and results with the rest of the collaborative. Collaboratives provide an infrastructure for an interorganisational support network from which members can address common barriers and learn from others' successes and challenges. ${ }^{6}$ The success of improvement collaboratives is determined by the influence of their interorganisational (shared learning) and intraorganisational features (culture, resources, leadership, etc). ${ }^{7}$

The evidence for the effectiveness of improvement collaboratives as interventions for improving health outcomes is growing but is not yet compelling. Indeed, collaboratives may have a greater impact on changes to professional behaviour and care processes than on care quality or health service outcomes. ${ }^{8}$ This is in part due to the heterogeneity of contexts within which collaboratives operate. ${ }^{9}$ Additionally, research studies have focused on demonstrating 'what' impact an improvement collaborative has had, overlooking 'why', 'how' and 'what works for whom in what context', with relatively few qualitative evaluations that can provide a rationale for their impact on outcomes. ${ }^{10}$

Studies have explored factors influencing clinician engagement in quality improvement but have not considered the role of motivation to participate, especially within a challenging healthcare environment with workforce problems such as low morale, increasing numbers of vacant clinical posts and issues with staff retention. ${ }^{11-13}$ Quality improvement initiatives often rely on the inherent motivation of practitioners to provide highquality care for their patients. ${ }^{14}$ Carter et $a l^{15}$ proposed that concepts such as 'collaborative advantage' emerging within a collaborative may affect motivation, instilling change through promoting competition among teams who may strive to attain the same level of advancement of others. Mixed empirical evidence for the effectiveness of improvement collaboratives coupled with minimal understanding of the motivation of practitioners to participate presents an important challenge to proponents of quality improvement approaches in healthcare.

\section{Participatory evaluation of a quality improvement collaborative}

Patient Safety Collaboratives

In 2014, NHS England commissioned 15 Patient Safety Collaboratives hosted by Academic Health Science Networks to deliver safety improvement in response to Professor Don Berwick's report A Promise to Learn-a commitment to act. ${ }^{16}$ This evaluation centres on an Academic Health Science Network with a partnership of NHS organisations (hospital trusts) in Southeast England, which identified sepsis and acute kidney injury (AKI) as key priority areas for patient safety because they were responsible for the greatest number of avoidable deaths in hospitalised patients. ${ }^{17}$ Using a Breakthrough Series approach, the Patient Safety Collaborative team aimed to improve patient outcomes through improving clinical process measures to enable rapid detection and treatment, using up-to-date and evidenced-based guidelines as well as building improvement capacity and capability in the participating hospital trust. As the host organisation, the Patient Safety Collaborative Team was responsible for the day-to-day operation of the collaborative.

\section{Participatory approach: the researcher-in-residence model}

Participatory approaches have the potential to close the gap between research and practice in improvement collaboratives by adopting a research-based approach to addressing the challenge of motivation of the participating teams and the individuals within them. Participatory approaches involve partnership with stakeholders to solve practical problems and a sustained commitment by researchers to continually collaborate. ${ }^{18}$ The in-residence model is an emerging model of participatory research which embraces the concept of 'cocreating' knowledge between researchers and practitioners, using a range of approaches. ${ }^{19}$ In this study, the researcher (ML) was embedded in the collaborative, acting as an interface between the emerging evidence from the evaluation and its application to collaborative processes, cocreating knowledge through participation, with research expertise communicated to and negotiated with the collaborative participants. The participatory approach was enhanced by the formation of an evaluation steering committee comprising the Patient Safety Collaborative team members, academic colleagues and clinical leads from hospital trusts in the collaborative. This committee codesigned and cointerpreted findings from the study, generating evidence that could potentially optimise motivation within the collaborative. This study explores the barriers and facilitators to motivation that may affect participation in an improvement collaborative using an illustrative case study and describes a practical approach to participatory evaluation.

\section{METHODS}

\section{Subjects and setting}

The collaborative ran from September 2015 to June 2017 with the evaluation undertaken between January 2016 and April 2017. Three teams participated in the evaluation: two were represented by patient safety teams from hospital trusts from the total collaborative membership of 23 teams and the third was the Patient Safety Collaborative programme team. The two teams representing the hospital trusts were purposively selected through discussions with the evaluation steering committee, based on their perceived contrasting maturity in terms of quality improvement capability and capacity. One team was well-resourced with a specific department dedicated to quality improvement in its hospital, while the second 
was viewed as similar to all other teams in the collaborative (limited resources for patient safety and minimal improvement expertise).

Overall, 15 individual semistructured interviews were conducted. An initial 13 interviews were held with team members of the two hospital trusts and the Patient Safety Collaborative team who were purposively selected based on their role in patient safety within their organisation. Using snowball sampling, a further two participants from the other collaborative teams were identified and interviewed..$^{20}$ Interview participants included six senior and middle managers, three doctors (two consultants and one registrar), five senior nurses (NHS band 7 and above) and one junior nurse (NHS band 5). There was no consistent patient representation in the teams and hence this group was not included in this study. As a result of the in-residence approach, all participants were known to the researcher and were aware of the purpose and aims of the study. Interview participants were approached in person or by email and there were no refusals to participate. Written informed consent was obtained from each participant prior to interview.

\section{Study design}

The evaluation was undertaken in a series of iterative stages of participation: data collection, analysis, interpretation and dissemination of emerging findings, with the application of evidence to influence the development of the programme. Qualitative methods were used to generate and analyse the data.

\section{Data collection}

Participant observation was undertaken by ML and involved attendance at trust and Patient Safety Collaborative meetings as well as collaborative learning sessions, totalling approximately 100 hours. As the evaluation progressed the researcher became an active participant in meetings, providing input and facilitating discussion. Field notes at meetings were recorded. Pertinent points arising from these observations were communicated to the teams to facilitate discussion at future meetings and to enable them to determine how to use the findings in relation to the progress of the collaborative.

Interviews were conducted by ML, a researcher with experience of conducting health service evaluations using qualitative methods. Interviews were held at the participant's workplace in a private meeting room and lasted between $45 \mathrm{~min}$ and $60 \mathrm{~min}$. Interviews were audio recorded and transcribed verbatim. No repeat interviews were carried out. The interview guide was based on the existing improvement collaborative literature and was informed in its development by participant observation data and documentary review as well as input from members of the evaluation committee. The guide was adapted iteratively using an inductive approach allowing the exploration of new emergent themes. Interviews ceased once thematic saturation was reached. A review of documents pertaining to the collaborative at trust level and those produced by the Patient Safety Collaborative team such as meeting notes was also undertaken. Documents were carefully scrutinised and emerging relevant themes were mapped to the thematic framework discussed in the data analysis section below.

\section{Data analysis}

Data were managed using NVivo V.11.0. ML conducted qualitative analysis using a thematic framework approach to code the data and identify patterns and themes. ${ }^{21}$ The framework was developed iteratively to capture emerging themes from the data and was also informed by field notes from participant observation. Components of the analysis plan such as the thematic framework were discussed with evaluation committee members, which facilitated their understanding of how their perceptions of the collaborative influenced their engagement with it. Cointerpretation of emerging themes with the evaluation committee was also conducted and alternative interpretations developing from these discussions were included in the analysis.

\section{RESULTS}

Two main categories of themes centred on the core concept of motivation emerged: (1) inherent motivation, relating to personal drivers and (2) factors that influence motivation, interorganisational and intraorganisational features common to improvement collaboratives as well as external factors.

\section{Inherent motivation (personal drivers)}

Inherent motivation at the level of the individual was integral to participation in the collaborative. Clinicians mentioned using the collaborative as an opportunity for career advancement and professional development. From collaborative learning sessions, it was observed that several of the patient safety teams focused on recruiting junior doctors, who were required to document evidence of quality improvement activities in their career portfolios. Additionally, some of the interviewees mentioned that witnessing the positive effect of improvement interventions provided a sense of personal achievement which acted as an enabler in motivating individuals.

Motivation was also shaped by individual attitudes and perceptions such as the extent of understanding of the purpose and value of quality improvement. Some clinicians suggested that quality improvement was not a priority and was regarded as secondary to the need to deliver routine care. Additionally, interviewees mentioned a degree of confusion among some of their staff about their understanding of the definition of quality improvement. Some described quality improvement as burdensome and unnecessary as it did not appear to directly benefit patients. Interviewees also mentioned using improvement methodology to focus on marginal gains, ensuring that new processes were beneficial to patient care with 
a preference for demonstrating small scale changes at a ward level.

It's about allowing people to try things out in a small environment... your tests of change through quality improvement are based on small scale steps of evidence, and if you can change something for just one person that must be a good thing. (Manager, partner trust)

As the collaborative progressed, a few 'champions' identified by the Patient Safety Collaborative team emerged. From learning sessions these champions were observed to be similar to change agents, driving the patient safety agenda in their teams and departments. Their motivation to shape the local level patient safety strategy and the sharing of this narrative with the collaborative led to the spread and adoption of a champion's model in other teams. Some champions emerged in trusts where there were significant internal and external pressures.

And then organisations that aren't necessarily in the good organisation space, they've really risen to the challenge and become involved, partly thanks to these individuals. (Patient Safety Collaborative team member)

\section{Factors affecting motivation}

\section{Intraorganisational features}

The most frequently mentioned or observed intraorganisational features that were potential barriers and facilitators to motivation were conflicting priorities, engagement and clinical ownership, and support and culture.

\section{Conflicting priorities}

At the time of this programme, patient safety teams in trusts from across the collaborative were allocating a significant proportion of their time to undertaking Commissioning for Quality and Innovation (CQUIN) data collection to meet financially incentivised targets. Interviewees felt that CQUIN detracted them from improving safety in the organisation, which ironically was the premise of the project.

... because our team spend so much time collecting data; reviewing patient records; trying to find information, they aren't using information to make a difference; through teaching; training; coaching... because, ultimately, CQUIN schemes earn this organisation about one and a half million a year. (Manager, partner trust)

\section{Engagement and clinical ownership}

Engagement and clinical ownership were key barriers to motivation at trust level. Interviewees mentioned insufficient support from some front-line clinicians, which was seen as limiting the embedding of quality improvement processes and affecting the motivation of team members. The lack of support was compounded by mainly senior staff undertaking improvement work with junior nurses in particular, neither aware or involved. These issues were thought to reflect a broader challenge of embedding an improvement culture within organisations.

... more could be done to raise awareness among the junior nurses about quality improvement. It seems to be something the consultants and senior nurses do. I think we should all be doing it or at least be aware of it or how will the whole organisation improve? (Nurse, partner trust)

In addition, one interviewee claimed that their organisation, despite previously having a good reputation for safety improvement, was now prioritising efficiency to ensure compliance with targets such as reducing accident and emergency waiting times. This efficiency drive was led by the Chief Executive Officer whose focus was on the operational aspects of the everyday running of the hospital.

The organisation is so operational. People should be thinking a bit more strategically and there should be discussions and planning as a joint effort. But actually the people at those levels, including the Chief Executive, spend a lot of time running the operations of the hospital. (Senior Manager, partner trust)

\section{Support and culture}

It was suggested that with fewer resources of time, financial support and staff with training in quality improvement, individuals responsible for implementing improvement projects were less motivated and that this may have affected their willingness to participate in the collaborative. Moreover, medical directors in some trusts did not involve clinicians in discussions about joining the collaborative and initially, some of these teams struggled to engage. Even so, as some interviewees mentioned, motivation could not be created, it had to exist within organisational culture. One of the collaborative organisations had a self-professed culture of improvement formed through a top-down approach resulting in a directorate of quality and a well-resourced patient safety team. Yet, even in this organisation, some expressed concerns about the extent of resource prioritisation for patient safety.

Interviewer: Why can't the funding gap be filled by the Board with resources from within the organisation?

Participant: Absolutely could be. But it's about showing that there's some return on investment. You know, Ophthalmology is a department that earns money. Maternity is a department that earns money. Patient Safety isn't a department that earns money. We save money. It's quite difficult. (Senior manager, partner trust)

\section{Interorganisational features}

Interviewees were generally positive about participating in the collaborative identifying interorganisational 
learning and sharing as key motivating factors to ongoing participation. Through observation of learning sessions, it was apparent that teams were proud of their achievements and showcasing these successes was an important incentive.

... we do not have many resources, but we have embraced learning and driven the patient safety work in our trust. I am sure others can do the same and if they come to appreciate that from seeing our achievements, that can only be a positive thing. (Nurse, partner trust)

Some interviewees suggested that the reputation of the collaborative was a significant reason for trusts to join. The Patient Safety Collaborative team members described their perceived successes and challenges relevant to ongoing motivation of the collaborative participants. Raising awareness of AKI and sepsis and information sharing across the collaborative through the use of emails, social media, webinars, teleconferences and learning sessions were viewed as a key success. Also mentioned, was facilitating the understanding of the benefits of sustained quality improvement approaches on health outcomes.

Sustaining engagement with the collaborative was viewed as a significant challenge and was thought to be because of intraorganisational issues such as staff turnover or dwindling resources. Patient Safety Collaborative team members also identified two key learnings: (1) in some cases, they had not fully explored and understood the factors affecting individual trust engagement and (2) they had not assessed the readiness of some organisations to effectively participate in the collaborative. Nonetheless, through observations it was apparent that even if reasons for less engagement were known, the Patient Safety Collaborative team struggled to achieve consensus on how best to support the relevant trust teams to overcome these factors, potentially affecting their motivation.

The team are unsure of the best strategy to deal with less engaged trusts. There are suggestions that discussions should be held with the MD who maybe able to facilitate engagement. Others feel that this could be counter-productive and that teams should be gently encouraged to participate and supported where possible to do so. (ML field notes).

It just highlights how you can't just brainwash an MD at a meeting and then rely on them for people to start coming and devolving time. So actually the Trusts that weren't performing or coming or engaging, I think that was a reflection of us. (Patient Safety Collaborative team member)

\section{External factors}

Two emerging subthemes relating to the health system (context and system pressures) that may have affected motivation were identified as external factors. The context within which the collaborative operated was similar in all trusts. For example, imminent or recent inspections by the national regulator, the Care Quality Commission (CQC) was frequently cited as a reason for joining the collaborative. There was also heightened awareness around sepsis due to a national campaign advocating for improving its recognition and treatment and significant changes to National Institute of Clinical Excellence guidelines. ${ }^{22}{ }^{23}$ Such high profile developments coupled with increased media scrutiny of several avoidable deaths ${ }^{24}$ were seen by interviewees as potential drivers for participation in the collaborative. Relevant to health system pressures, interviewees from the trusts expressed an ambivalence to minimal resource provision for patient safety, acknowledging that working within tight parameters was the norm within the context of the current healthcare environment in the UK, yet, participating in quality improvement was still possible.

You have to look at what resources you've got, and then how you can do what you need to within that.... in this climate, and in this organisation, I think we should be realistic, we have to work differently to achieve outcomes. (Doctor, partner trust)

\section{DISCUSSION}

The study has revealed three principal findings that provide important lessons for quality improvement programmes in the NHS. First, individuals can act as change agents, driving the quality improvement agenda at a local level. Second, inadequate board (medical director) engagement at trust level may affect motivation among team members. Finally, improvement collaboratives may have a key role in the UK healthcare system, as they promote shared learning and the formation of networks that are established on the premise of mutual experience and a common purpose. Following the completion of this collaborative programme, a deteriorating patient community of practice was formed as well as a focus on paediatric sepsis across the partner trusts in London. This is one such example of the potential legacy of improvement collaboratives.

A strength of this study was that the participatory approach to the evaluation made a positive contribution to the progression of the collaborative in terms of: the provision of operational support, mobilisation of current knowledge (from the academic literature) as well as sharing newly generated knowledge to assist the programme in meeting its objectives and the sharing of information to connect different components of the programme. An in-depth focus on two trust teams was a trade-off for more generalisable findings. Nonetheless, one of these teams was representative of most of the collaborative members in terms of its intraorganisational features. Moreover, the in-depth participatory approach enabled the researcher to generate findings and insights on the motivation of individuals within a patient safety team which may be transferable to similar settings in NHS trusts. An additional limitation was that of the in-residence model whereby sometimes it was 
challenging for the researcher to maintain objectivity with fellow team members as participants in the evaluation. This was minimised by the researcher regularly discussing findings with independent academic colleagues. Furthermore, participatory approaches acknowledge the importance of including individuals familiar with a programme or service, providing a context for findings and enabling learning through the process of evaluation. ${ }^{25}$

Most studies in this field have used a summative approach to evaluate the effectiveness of an improvement collaborative as an intervention to improve patient outcomes. In contrast, this study provides a novel approach, using a qualitative and formative evaluation with a researcher embedded in an improvement collaborative. This approach has revealed some key aspects of the 'black box' that exists in terms of understanding 'how' and 'why' a collaborative is effective through focussing on motivation at the level of the individual and organisation. Yet, it has not established the effect of motivation within this Patient Safety Collaborative on patient outcomes for AKI and/or sepsis.

Three key aspects of embedded research identified from a recent review of the role of embedded research in quality improvement programmes ${ }^{26}$ have been identified as relevant to this evaluation: (1) the researcher became a key member of the Patient Safety Collaborative team playing an operational and evaluative role, (2) important working relationships with staff were developed and (3) knowledge was coproduced through partnership working with the evaluation committee. These aspects were especially pertinent to an embedded researcher working as a member of an organisation in an improvement collaborative whereby the implementation of the improvement methodology and its perceived impact required 'real-time' feedback from collaborative members to enable the host team to adapt and improve its processes, directed toward encouraging the teams to learn and share from each other. In this study, we have highlighted some of the experiences of an in-residence researcher in a service or programme that have also been described elsewhere. ${ }^{27}$ However, it is challenging to fully assess and attribute change to the role of the researcher on the progress of this collaborative and its outcomes. There is scope for further exploration of this aspect in future studies using the researcher-in-residence model.

This study has important implications for clinicians and managers planning and implementing quality improvement programmes in NHS organisations as well for those agencies involved in hosting such initiatives. At the individual level, career advancement and personal achievement provide organisations with a focus for incentivising clinician participation in quality improvement as is the case with junior doctors in this evaluation whose recruitment to such initiatives may enhance organisational systems and develop medical professionalism. ${ }^{28}$ The individual perception of the term 'quality improvement' also influenced motivation and caused confusion among some, acting as a potential barrier to motivation. The evaluation suggests that reframing quality improvement for clinicians, as a series of marginal gains, whereby the immediate discernible and small-scale benefits are perceived as acceptable, may encourage participation in improvement programmes. It is rare to achieve the dramatic scale of improvement seen in the much cited 'Matching Michigan' study. ${ }^{29}$

The findings also highlight important external factors (over which the collaborative teams had little control) that influence motivation and the ability of teams to engage, potentially negatively affecting other members of the collaborative network. ${ }^{30}$ Research has suggested that existing organisational culture can be superseded by sufficiently empowered managers and clinicians, similar to the champions of this collaborative, who emerged from trusts where an improvement culture was not apparent. ${ }^{31}$ Armenakis et al suggest that to institutionalise permanent change, change agents require credibility within their organisation. ${ }^{32}$ In this collaborative, 'champions' were senior clinical staff who had demonstrated a desire to lead an improvement programme. The Patient Safety Collaborative team successfully harnessed the inherent motivation of these individuals resulting in spread and adoption of several 'champions' inspired approaches to patient safety across the collaborative.

The 'all share, all learn' approach of the Patient Safety Collaborative team has revealed some of the potential benefits of a non-hierarchical network, such as promoting togetherness among teams, providing reassurance, trust and a common purpose, ${ }^{33}$ which is especially pertinent at a time when pervasive health system pressures are affecting workforce motivation. ${ }^{34}$ The study findings suggest that the collaborative was integral in raising awareness and facilitating the improvement of local level clinical processes for AKI and sepsis. The reputation of the Patient Safety Collaborative host organisation was identified as a means of gaining recognition with trust boards, the CQC and other clinicians. Hence, there is a potential gap for improvement collaboratives to bridge in the healthcare system in England, in building capability and capacity and motivating individuals to participate in quality improvement, especially in organisations that have neither the resource or volition.

The NHS trusts in this improvement collaborative demonstrated different levels of readiness despite facing similar challenges relating to their intraorganisational features. This was in part associated with the engagement of trust boards, which demonstrated an initial interest in participating in the collaborative by signing a contract agreement with the host organisation, but, in some cases this failed to develop into a sustained commitment and may have been overtaken by other priorities. Using a bottom-up approach, involving front-line clinicians from the outset, may facilitate the continued engagement of organisations in an improvement collaborative. Additionally, other intraorganisational features such as a lack of support and resources are prominent in this evaluation and affect motivation. Nevertheless, some interviewees mentioned that carrying out quality improvement work in addition to providing routine care within existing resource parameters was acceptable and achievable. A recent King's fund report suggested that trusts and individuals should be pursing approaches to 
continuously improve the quality of care despite a lack of resources. $^{35}$

The host organisation in this improvement collaborative is one of several Academic Health Science Networks across England involved in building improvement capacity and capability within their partnership of NHS trusts. This raises a question of whether it is the responsibility of individual NHS organisations themselves to allocate resources for improvement initiatives or whether it is the role of an external organisation. The host organisation's role is to develop programme aims, disease-specific measures and tools as well as to facilitate and provide technical support but in this study, they acted as a proxy quality improvement team for some of the NHS organisations. This is a significant challenge for external organisations as they try to find a role within a healthcare system while acting as enablers for their partners. This study should initiate further discussion and examination of the role of organisations such as Academic Health Science Networks that may provide significant support for quality improvement to individual NHS trusts.

Acknowledgements The authors thank all of the study participants and in particular the two trusts that allowed the researcher to be embedded within their patient safety teams as well as the contribution of several of the Patient Safety Collaborative team members through the course of the evaluation. The authors also acknowledge the contribution of Professor Roger Jones who provided guidance on some aspects of the structure and framing of the manuscript.

Contributors $\mathrm{ML}, \mathrm{MM}, \mathrm{KH}$ and $\mathrm{MS}$ conceived and planned the overall study. ML, $\mathrm{MM}$ and MS wrote the initial study protocol. ML, MM, KH, MS, CL, JW, LE and PT codesigned aspects of the study including the strategy for sampling of the two trusts for participation in the evaluation and reviewing tools for data collection. $M L$ undertook recruitment, data collection and analysis. All the authors formed the overall membership of the evaluation steering committee, cointerpreted the study findings, read and approved the final version of the manuscript. ML drafted the manuscript and revised it in response to comments from all coauthors.

Funding This work was supported by NHS England (grant no: 533957). NHS England provided the funds to UCLPartners to host a Patient Safety Collaborative of which some were allocated to undertaking this evaluation.

Competing interests None declared.

Patient consent Not required.

Ethics approval Ethics approval was obtained from the University College London Research Ethics Committee (reference no 16/0007).

Provenance and peer review Not commissioned; externally peer reviewed. Data sharing statement № additional data are available.

Open Access This is an Open Access article distributed in accordance with the Creative Commons Attribution Non Commercial (CC BY-NC 4.0) license, which permits others to distribute, remix, adapt, build upon this work non-commercially, and license their derivative works on different terms, provided the original work is properly cited and the use is non-commercial. See: http://creativecommons.org/ licenses/by-nc/4.0/

(C) Article author(s) (or their employer(s) unless otherwise stated in the text of the article) 2018. All rights reserved. No commercial use is permitted unless otherwise expressly granted.

\section{REFERENCES}

1. ØVretveit J, Bate P, Cleary P, et al. Quality collaboratives: lessons from research. Qual Saf Health Care 2002;11:345-51.

2. Power M, Tyrrell PJ, Rudd AG, et al. Did a quality improvement collaborative make stroke care better? A cluster randomized trial. Implement Sci 2014;9:40.
3. Marshall M, Mountford J, Gamet K, et al. Understanding quality improvement at scale in general practice: a qualitative evaluation of a COPD improvement programme. Br J Gen Pract 2014;64:e745-e751.

4. Working together to make all our lives better: Collaborative care in residential homes: The Health Foundation, 2017.

5. The breakthrough series: IHI's collaborative model for achieving breakthrough improvement: Innovation Series: Institute for Healthcare Improvement, 2003.

6. Nadeem E, Olin SS, Hill LC, et al. Understanding the components of quality improvement collaboratives: a systematic literature review. Milbank Q 2013;91:354-94.

7. Nembhard IM. Learning and improving in quality improvement collaboratives: which collaborative features do participants value most? Health Serv Res 2009;44:359-78.

8. De Silva D. Improvement collaboratives in health care. London, UK: The Health Foundation, 2014.

9. Schouten LM, Hulscher ME, van Everdingen JJ, et al. Evidence for the impact of quality improvement collaboratives: systematic review. BMJ 2008;336:1491-4.

10. Howe C, Randall K, Chalkley S, et al. Supporting improvement in a quality collaborative. British Journal of Healthcare Management 2013;19:434-42.

11. Ling T. How do you get clinicians involved in quality improvement?: an evaluation of the health foundation's engaging with quality initiative: a programme of work to support clinicians to drive forward quality: final report: The Health Foundation, 2010.

12. Davies H, Powell A, Rushmer R. Why don't clinicians engage with quality improvement? J Health Serv Res Policy 2007:12:129-30.

13. lacobucci G. Future gaps in workforce pose an impending crisis for the NHS, report warns. BMJ 2013;347:f4696.

14. Dixon-Woods M, McNicol S, Martin G. Ten challenges in improving quality in healthcare: lessons from the Health Foundation's programme evaluations and relevant literature. BMJ Qual Saf 2012;21:876-84

15. Carter P, Ozieranski P, McNicol S, et al. How collaborative are quality improvement collaboratives: a qualitative study in stroke care. Implement Sci 2014;9:1-11.

16. Berwick D. A promise to learn - a commitment to act: improving the safety of patients in England. London, England: NHS, 2013.

17. Patient Safety: The UCLPartners Patient Safety Programme. Secondary Patient Safety: The UCLPartners Patient Safety Programme 2017. http://uclpartners.com/what-we-do/patientsafety/.

18. Eyre L, Farrelly M, Marshall M. What can a participatory approach to evaluation contribute to the field of integrated care? BMJ Qual Saf 2017;26:588-94.

19. Marshall M, Pagel $\mathrm{C}$, French $\mathrm{C}$, et al. Moving improvement research closer to practice: the Researcher-in-Residence model. BMJ Qual Saf 2014;23:801-5. bmjqs-2013-002779.

20. Palinkas LA, Horwitz SM, Green CA, et al. Purposeful Sampling for Qualitative Data Collection and Analysis in Mixed Method Implementation Research. Adm Policy Ment Health 2015;42:533-44

21. Gale NK, Heath G, Cameron E, et al. Using the framework method for the analysis of qualitative data in multi-disciplinary health research. BMC Med Res Methodol 2013;13:117.

22. Freitag A, Constanti M, O'Flynn N, et al. Guideline Development Group. Suspected sepsis: summary of NICE guidance. BMJ 2016;354:i4030.

23. Wise J. Sepsis should be treated within one hour, says NICE. BMJ 2017;356:j1257.

24. Rawlinson K. Spotting sepsis 'could prevent 37000 deaths a year. The Guardian 2016.

25. Griffin S, Glover SH, Williams AW, et al. Participatory evaluation of community-based HPV and cervical cancer prevention and control efforts. J S C Med Assoc 2009;105:309-17.

26. Vindrola-Padros C, Pape T, Utley M, et al. The role of embedded research in quality improvement: a narrative review. BMJ Qual Saf 2017;26:70-80

27. Marshall M, Eyre L, Lalani M, et al. Increasing the impact of health services research on service improvement: the researcher-inresidence model. J R Soc Med 2016;109:220-5.

28. Involving junior doctors in quality improvement London. UK: The Health Foundation, 2011.

29. Bion J, Richardson A, Hibbert P, et al. Matching Michigan Collaboration \& Writing Committee. 'Matching Michigan': a 2-year stepped interventional programme to minimise central venous catheter-blood stream infections in intensive care units in England. BMJ Qual Saf 2013;22:110-23.

30. Aveling EL, Martin G, Herbert G, et al. Optimising the communitybased approach to healthcare improvement: Comparative case 
studies of the clinical community model in practice. Soc Sci Med 2017;173:96-103.

31. Phung VH, Essam N, Asghar Z, et al. Exploration of contextual factors in a successful quality improvement collaborative in English ambulance services: cross-sectional survey. J Eval Clin Pract 2016;22:77-85.

32. Armenakis AA, Harris SG, Feild HS. Making change permanent a model for institutionalizing change interventions. Research in organizational change and development: Emerald Group Publishing Limited, 2000:97-128.

33. Health leaders' panel survey 6: footprints, financing and staff morale: Nuffield Trust, 2015

34. Huxham C, Vangen S. Managing to collaborate: the theory and practice of collaborative advantage. Abingdon: Routledge, 2013.

35. Dawda P, Jenkins R, Varnam R. Quality improvement in general practice: The King's Fund, 2010. 